\title{
Not your typical back pain: A case report of reactive arthritis
}

\author{
Irfan Chhipa $^{1^{*}}$, Quincy Cheesman ${ }^{2}$ \\ ${ }^{1} \mathrm{MD},{ }^{2} \mathrm{DO},{ }^{1}$ Rothman Orthopaedic Institute, Egg Harbor Township NJ, United States, ${ }^{2}$ Rothman Orthopaedic Institute, Philadelphia, PA, \\ United States
}

*Corresponding Author: Irfan Chhipa

Email: irfan.chhipa@rothmanortho.com

\begin{abstract}
Introduction: Reactive arthritis classically includes a triad of conjunctivitis, urethritis, and arthritis in a young, adult male patient following a urogenital or gastrointestinal infection. This paper reports a case of reactive arthritis in a young, sexually active, male patient who presents atypically, with a chief complaint of three-month lower back pain.

Case Report: A 30-year-old male presents to the office with a complaint of three-month lower back pain despite conservative treatment and a negative workup in the emergency department, two months prior. After a thorough assessment and laboratory workup, the patient was diagnosed and treated for reactive arthritis. Suspicion for reactive arthritis included a positive Chlamydia trachomatis urinalysis and a positive HLA-B27. Three weeks after appropriate treatment, the patient is now symptom-free.

Conclusion: In conclusion, it is important to thoroughly assess a patient who presents with continued pain despite previous workup and treatment. Lower back pain is a common diagnosis and therefore other possible diagnoses are often times written off. Establishing the correct diagnosis may require thinking outside of the box and obtaining multiple labs. However, getting the correct diagnosis is of utmost importance for both the patient and medical community so that further complications and progression of disease do not occur.
\end{abstract}

Keywords: Reactive arthritis, Chlamydia trachomatis, Young male, Sexually active, Classic triad.

\section{Introduction}

Reactive arthritis is a seronegative multisystem inflammatory disorder classically involving the joints, the eyes, and the lower genitourinary tract. This classically includes the triad of conjunctivitis, urethritis, and arthritis in young patients following a urogenital or gastrointestinal infection. ${ }^{1}$ It is well known to medical students as the syndrome where one " can't see, can't pee, and can't climb a tree." Additionally, there may be spine or dermatologic manifestations.

Several bacteria, such as Salmonella, Shigella, Yersinia, Campylobacter, and Chlamydia species have been strongly implicated as a triggering factor for reactive arthritis. ${ }^{2}$ With this being said, infection with Chlamydia trachomatis, which is a common sexually transmitted bacterial disease, has emerged as a major causative agent of reactive arthritis. ${ }^{3}$ Several studies involving reactive arthritis patients have shown evidence of present or prior chlamydia infection. ${ }^{4}$ Additionally, there appears to be a genetic relationship amongst the majority of patients with reactive arthritis. Approximately $80 \%$ of patients are HLA-B27 positive. ${ }^{5}$ Despite the current knowledge on the etiology of reactive arthritis, the pathogenesis is not completely understood. There is theory of cross-reactivity between HLA-B27 and causative bacteria. However, there is no strong supporting evidence to support this theory.

This paper reports a case of reactive arthritis in a 30year-old, sexually active male. The purpose of reporting this case is to exemplify a clinical presentation of reactive arthritis in order to enlighten physicians on additional differential diagnosis when working up a patient with similar symptoms.

\section{Case Report}

A 30-year old male, with no significant past medical history, presents to the outpatient office for evaluation of threemonth, persistent, lower back pain. The patient is a recreational athlete who plays basketball and football. He had been seen in the emergency room two months prior with the same lower back pain. Radiographs of the lumbar spine were unremarkable. At that time, he was diagnosed with a lower back strain and discharged home with NSAIDs and a muscle relaxant.

Three months from the onset of pain, he presents to the office localizing pain to the lower back. He describes it as sharp and non-radiating with a pain scale of $8 / 10$. The pain prevents him from getting adequate sleep at night. It is exacerbated with movement in any direction and is minimally alleviated with NSAIDs. The patient does report of associated morning stiffness. He does not recall any trauma, nor any injuries during his recreational activities. He denies any weakness or numbness/tingling in his lower extremities, buttock pain, bowel/bladder incontinence, pain/swelling in any other joints/tendons, any recent infections, diarrhea, urinary symptoms, urethral discharge, fever, or IV drug abuse. The patient does admit to being sexually active with multiple partners and does not use condoms.

Physical exam revealed a well-developed, wellnourished, Caucasian, middle-aged male in moderate distress. The patient's vital signs were all within normal limits and he was able to ambulate into the office without much difficulty. He had tenderness to palpation along the posterior lumbar region with limited range of motion secondary to pain, more so with flexion. There were no signs of swelling or erythema. His muscle strength was $5 / 5$ in bilateral lower extremities with sensation intact to light touch throughout. There were no significant gait abnormalities, eye erythema, eye discharge, oral lesion, murmur, or other joint involvement.

Considering the clinical picture and physical exam, the differential diagnosis included lumbar strain, herniated disk, spondylolisthesis, spondylolysis, spondylosis, acute 
monoarthritis, rheumatoid arthritis, ankylosing spondylitis, infection, or malignancy.

Laboratory investigation revealed a white blood cell count of $5,700 \mathrm{~mm}^{3}\left(4,500 \mathrm{~mm}^{3}-11,000 \mathrm{~mm}^{3}\right)$, hemoglobin of $14.7 \mathrm{~g} / \mathrm{dL}(13.5 \mathrm{~g} / \mathrm{dL}-17.5 \mathrm{~g} / \mathrm{dL})$, hematocrit of $44.9 \%$ (41\%$53 \%)$, platelets of $187,000 \mathrm{~mm}^{3}\left(150,000 \mathrm{~mm}^{3}-400,000 \mathrm{~mm}^{3}\right)$, erythrocyte sedimentation rate (ESR) of $16 \mathrm{~mm} / \mathrm{h}(0 \mathrm{~mm} / \mathrm{h}$ $15 \mathrm{~mm} / \mathrm{h}$ ), and alkaline phosphatase (ALP) of $56 \mathrm{IU} / \mathrm{L}$ (44IU/L-147IU/L). Additionally, urinalysis revealed a white blood cell count of $2-5 / \mathrm{hpf}$, occasional bacteria, nitrite negative, leukocyte esterase negative, Chlamydia trachomatis positive, and Neisseria gonorrhoeae negative. Other labs revealed HIV-1/HIV-2 screen non-reactive, RPR non-reactive, stool cultures negative, HLA-B27 positive, ANA negative, and Rheumatoid factor negative. Radiographic imaging of the lumbar spine, done in the office, revealed no abnormalities (Fig. 1).

Based on the presence of persistent lower back pain and elevated inflammatory markers, such as ESR, the patient had HLA-B27 testing conducted to evaluate for rheumatological causes of his symptoms. It was also found that the patient had a history of multiple sexual encounters without protection, which prompted a workup for sexually transmitted diseases. Results indicated a positive Chlamydia trachomatis test. Based off of the patient's symptoms and laboratory results, he was ultimately diagnosed with reactive arthritis.

The patient was treated with Doxycycline $100 \mathrm{mg}$ PO BID for 14 days and Indomethacin $50 \mathrm{mg}$ PO TID. He was also counseled on safe sex practices and food safety. The patient was compliant with the treatment and tolerated it well without any complications. Fortunately, he became completely pain-free three weeks after initiation of treatment. Additionally, the patient underwent a short course of physical therapy and returned to full activity. No further routine follow-up was warranted.
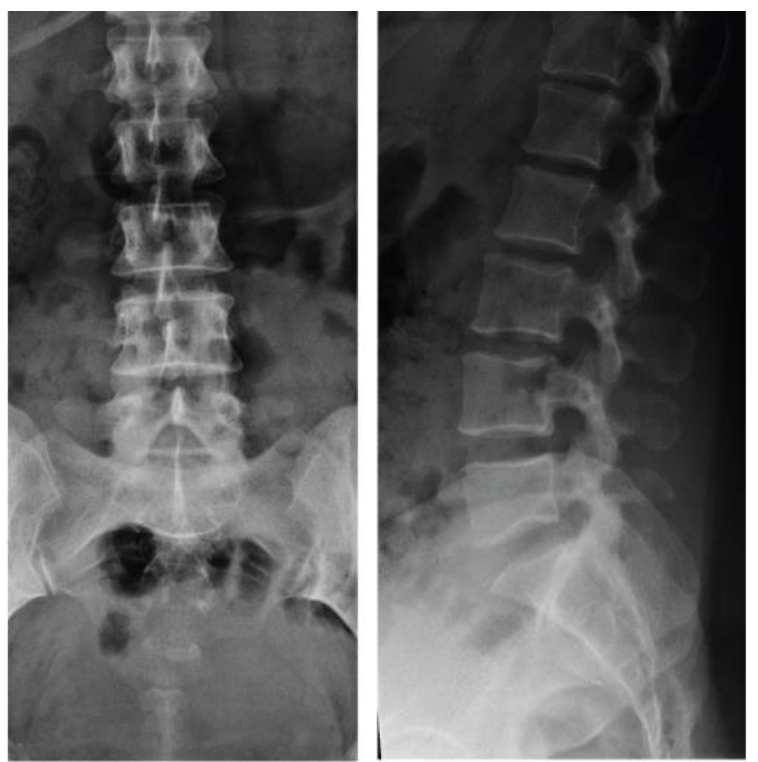

Fig. 1: AP and lateral radiographs of the patient's lumbar spine demonstrating no obvious abnormalities.
Percent sensitivity and specificity of various criteria for typical Reiter's syndrome*

\begin{tabular}{lcc}
\hline \multicolumn{1}{c}{ Method of classification } & Sensitivity & Specificityt \\
\hline $\begin{array}{l}\text { 1. Episode of arthritis of more } \\
\text { than 1 month with urethritis } \\
\text { and/or cervicitis }\end{array}$ & $\begin{array}{c}84.3 \% \\
(70 / 83)\end{array}$ & $\begin{array}{c}98.2 \% \\
(163 / 166)\end{array}$ \\
$\begin{array}{l}\text { 2. Episode of arthritis of more } \\
\text { than 1 month and either } \\
\text { urethritis or cervicitis, or } \\
\text { bilateral conjunctivitis }\end{array}$ & $\begin{array}{c}85.5 \% \\
(71 / 83)\end{array}$ & $\begin{array}{c}96.4 \% \\
(160 / 166)\end{array}$ \\
$\begin{array}{l}\text { 3. Episode of arthritis, con- } \\
\text { junctivitis, and urethritis }\end{array}$ & $\begin{array}{c}50.6 \% \\
(42 / 83)\end{array}$ & $\begin{array}{c}98.8 \% \\
(164 / 166)\end{array}$ \\
$\begin{array}{l}\text { 4. Episode of arthritis of more } \\
\text { than 1 month, conjunctivitis, }\end{array}$ & $\begin{array}{c}48.2 \% \\
\text { and urethritis }\end{array}$ & $\begin{array}{l}98.8 \% \\
(40 / 83)\end{array}$ \\
\hline
\end{tabular}

- Initial episode.

$\dagger$ Numbers in parentheses indicate number of patients correctly classified/number tested.

Fig. 2: Chart of percent sensitivity \& specificity of various criteria for typical reactive arthritis.

\section{Discussion}

Reactive arthritis is an inflammatory arthritis that occurs as a consequence of infection at a remote site. It should be suspected when there is a recent history of gastrointestinal or genitourinary infection. The clinical triad includes arthritis, urethritis, and conjunctivitis. A number of other clinical features have been observed to be associated with reactive arthritis including, but not limited to, axial arthritis, peripheral arthritis, low back pain, dactylitis, enthesitis, genitourinary tract symptoms, gastroenteritis, oral ulcers, balanitis, aortitis, and dermatologic manifestations. Despite knowledge of these clinical symptoms, they do not develop until 1-4 weeks after the initial infection. Further, these symptoms are not always clinically apparent. ${ }^{6}$

The etiology of reactive arthritis is still not completely understood. Particular infections and genetic factors have a very strong correlation with the development of reactive arthritis. ${ }^{7}$ It is believed that reactive arthritis is precipitated by infectious etiologies, specifically gastrointestinal or genitourinary infections. The genitourinary form is mainly caused by Chlamydia trachomatis while the gastrointestinal form is caused by Salmonella, Campylobacter, Shigella, or Yersinia. ${ }^{8}$

Additionally, patients with reactive arthritis also appear to have a strong genetic disposition with HLA-B27. The HLA-B27 antigen was found in $65-80 \%$ of patients with reactive arthritis. In $40-50 \%$ of cases of chlamydial infection, the HLA-B27 antigen was present. ${ }^{9}$ The importance of the HLA-B27 antigen comes into play when discussing the theory of cross-reactivity between the infectious etiological bacteria and HLA-B27 as the underlying pathogenesis for reactive arthritis. Despite the abundant knowledge of the disease, the pathogenesis is still not completely understood.

Generally, the diagnosis of reactive arthritis is clinical, given there are not any definitive diagnostic laboratory test or radiographic findings to establish the diagnosis. The American College of Rheumatology published a chart displaying the percent sensitivity and specificity of various 
criteria for typical reactive arthritis (Fig. 2). ${ }^{10}$ Laboratory testing is generally used to exclude other causes of arthritis and to further confirm any infectious cause. Laboratory testing in favor of a reactive arthritis diagnosis include elevated inflammatory markers (ESR \& CRP), elevated white blood cell count, elevated urinalysis white blood cell count, positive urinalysis cultures, and positive HLA-B27. Regardless of these findings, other laboratory results and imaging modalities are important to obtain in order to rule out additional causes such as rheumatoid arthritis, herniated disc, ankylosing spondylitis, and many more.

The patient, in this case, presented to the outpatient office with three months of persistent lower back pain despite treatment and a negative workup in the emergency department, two months prior. Given this information, it was important to create a broad differential diagnosis when working up the patient in order to prevent missing the diagnosis. It would be easy to neglect reactive arthritis in the differential diagnosis given our patient's lack of the "clinical triad" and the syndromes low incidence in comparison to other common causes. However, given the patient's sexual history and persistent pain, it is important to assess for reactive arthritis due to its strong association with Chlamydia trachomatis, a common sexually transmitted disease.

The patient's sexual history, positive Chlamydia trachomatis urinalysis, and positive HLA-B27 pointed towards the diagnosis of reactive arthritis as the cause of our patient's persistent, lower back pain. Other lab findings and imaging were able to rule out other causes including spondylolisthesis, spondylolysis, spondylosis, acute monoarthritis, rheumatoid arthritis, ankylosing spondylitis, infection, and malignancy.

Despite the fact that our patient did not present with the typical clinical triad, his symptoms seemed to fit the diagnosis and was treated as such. Treatment for the patient included Doxycycline $100 \mathrm{mg}$ PO BID for 14 days, Indomethacin $50 \mathrm{mg}$ PO TID, and formal physical therapy. When treating, it is important to treat both the patient and their partners to decrease the risk of subsequent arthritis. Other treatment options include Azithromycin $1 \mathrm{~g}$ orally as a single dose, Doxycycline $100 \mathrm{mg}$ PO BID for 7 days, Indomethacin 25-50mg PO TID for pain control, and corticosteroid injections. Physical therapy can also be done to improve mobility, strength, and prevent stiffness. Patients with persistent symptoms, greater than 3-6 months, can be treated with disease-modifying antirheumatic drugs (DMARDs) such as Sulfasalazine (1g PO BID). ${ }^{11}$

The patient's symptoms completely improved three weeks after initiating treatment. In regards to his prognosis, $10 \%-20 \%$ of patients with successfully treated reactive arthritis may continue to have chronic, destructive, and disabling arthritis two years after the onset of symptoms. ${ }^{12}$ Most likely, he will remain disease-free with no further sequelae. However, it is important to educate on safe sex practices to prevent another recurrence.

\section{Conclusion}

In conclusion, we presented a case involving a sexually active, 30-year-old male presenting with three months of persistent lower back pain despite conservative treatment modalities and a negative emergency room workup. The patient was ultimately diagnosed and treated, in the outpatient clinic, with reactive arthritis given his suspicious history and appropriate laboratory findings. The purpose of presenting this case is to educate other physicians on a unique clinical presentation of reactive arthritis.

The patient did not present with the typical clinical triad of reactive arthritis; however, persistent pain led us to believe there was something more going on which initiated an extensive workup. Without the audacity of conducting a broad workup, we may have missed the diagnosis resulting in continued pain, multiple doctor visits, and further sequelae of the disease.

In general, lower back pain is very common in the medical community and is typically attributed to a common diagnosis such as a muscle strain. Other less common causes of lower back pain are easily written off leading to a delay in the true diagnosis. We recommend that anytime a patient's symptoms do not make sense, an extensive and thorough workup should be conducted to prevent missing a diagnosis that can lead to worsening outcomes for both the patient and medical community.

\section{Written Disclosures}

The author of this paper has no potential conflicts of interest relating to, directly or indirectly, the subject of this case report.

\section{References}

1. Wu IB, Schwartz RA. Reactive Arthritis: The classic triad and more. Journal of the American Academy of Dermatology. 2008;59(1):113-121. doi:10.1016/j.jaad.2008.02.047

2. Department of Experimental Medicine, Section of Microbiology and Clinical Microbiology, Faculty of Medicine and Surgery-Second University of Naples, Italy and Department of General Pathology, Faculty of Medicine and Surgery-Second University of Naples, Naples, Italy.

3. Martin DH, Pollock S, Kuo CC, Wang SP, Brunham RC, Holmes KK. Chlamydia trachomatis infections in men with Reactive Arthritis. Ann Intern Med 100: 207-213, 1984.

4. Keat A, Thomas B, Hughes R, Taylor-Robinson D. Chlamydia trachomatis in reactive arthritis. Rheumatol Int 1989;9:197200.

5. Al-Khonizy W, Reveille JD. The immunogenetics of the seronegative spondyloarthropathies. Baillieres Clin Rheumatol 1998;12:567-88.

6. Koga T, Miyashita T, Watanabe T. Reactive Arthritis which Occurred One Year After Acute Chlamydial Urethritis. Intern Med 2008;47(7):663-6. doi:10.2169/internalmedicine.47.0581

7. Yu D, Kuipers JG. Role of bacteria and HLA-B27 in the pathogenesis of reactive arthritis. Rheum Dis Clin North Am 2003;29:21-36.

8. Koga T, Miyashita T, Watanabe T. Reactive Arthritis which Occurred One Year After Acute Chlamydial Urethritis. Intern Med 2008;47(7):663-6. doi:10.2169/internalmedicine.47.0581

9. Kwiatkowska B, Filipowicz-Sosnowska A. Reactive arthritis. Pol Arch Med Wewn 2009;119:60-5. 
10. Willkens RF, Arnett FC, Bitter T. Reactive Arthritis. Evaluation of preliminary criteria for definite disease. Bulletin Rheumatic Diseases 1982;32(4):31-4.

11. Barber CE, Kim J, Inman RD, Esdaile JM, James MT. Antibiotics for Treatment of Reactive Arthritis: A Systematic Review and Metaanalysis. J Rheumatol 2013;40(6):916-28. doi:10.3899/jrheum.121192

12. Not Your Typical Low Back Pain. American Medical Society for Sports Medicine. Retrieved from https://www.amssm.org/not_your_typical_low_back_csa115.html?StartPos=250\&Part=4

How to cite this article: Chhipa I, Cheesman Q. Not your typical back pain: A case report of reactive arthritis. Int $J$ Orthop Rheumatol 2019;5(2):70-3. 